\title{
Malignant Vulvar Phyllodes Tumor
}

National Cancer Institute

\section{Source}

National Cancer Institute. Malignant Vulvar Phyllodes Tumor. NCI Thesaurus. Code C128165.

A low or high grade malignant phyllodes tumor arising from specialized anogenital glands. 